\title{
Chapter 8 \\ Interspecific Interactions: Interaction \\ Modes Between Sound and Movement \\ in Collaborative Performance
}

\author{
Manoli Moriaty
}

\begin{abstract}
Collaboration between composers and choreographers is an approach possessing a long history and expansive list of creative outcomes. In recent years, such collaborative endeavours have further engaged with knowledge and practices from scientific disciplines, consequently giving rise to an emergent field of research focusing on novel modes of interaction between the expressive media of sound and movement as facilitated by new technologies and methodologies; from analogue proximity devices permitting dancers rudimentary control over recordings in the 1965 Variations $V$, to Marco Donnarumma utilizing biophysical sensors and machine learning algorithms as means of maintaining detailed command of digital signal processing (DSP) through specific body postures in the 2016 Corpus Nil. Whilst the contributions by this relatively new transdisciplinary field have produced practice and research outcomes of unquestionable value, its main focus concentrates on the expression of sound through movement, with lesser emphasis placed on perspectives of practitioners utilizing interactive sound as means of informing the arrangement of movement. This chapter aims to reflect those perspectives through the research conducted during my collaborations with dance practitioners, where we have together examined areas such as each performer's role while operating interactive systems, employing different modes of system operation according to the desired determinacy of resulting material, and simplification of discipline-specific language when engaged in polydisciplinary collaboration. The contribution of this research concerns a novel taxonomy of interaction modes informed by the biological phenomenon of symbiosis. Defined as close and persistent relationships between organisms of different species, symbiosis manifests in three core types-mutualism, commensalism, and parasitism - with each type determined according to the change in fitness outcome experienced by each of the engaged organisms. In the context of my collaborative practice, the biological notion of fitness outcome is interpreted as the expressive range allocated to each performer and their respective media, while simultaneously relating to the level of determinacy from the score and choreography. The symbiotic modes of interaction are firstly described through examining three contemporary precedents, each showcasing distinct approaches in
\end{abstract}

M. Moriaty ( $\varangle)$

University of Salford, Salford, UK

e-mail: e.moraitis1@salford.ac.uk

(C) The Author(s) 2020

R. Earnshaw et al. (eds.), Technology, Design and the Arts-Opportunities and Challenges, Springer Series on Cultural Computing, https://doi.org/10.1007/978-3-030-42097-0_8 
collaboration and use of gesture recognition technologies (GRT), followed by a practical demonstration of each mode by its activation during a practice outcome resulting from my collaborations with two dancers, including a detailed analysis of the developed interactive system and its principles of its operation.

Keywords Polydisciplinary $\cdot$ Collaboration $\cdot$ GRT $\cdot$ Interaction $\cdot$ User interface • Symbiosis

\subsection{Introduction}

For the better part of the past decade, a significant portion of my practice and research has been concerned with polydisciplinary (see note 1) collaboration in performance practice, with a particular focus on the processes by which practitioners expressing through sound and movement engage in collaboration. Since its early conception, my approach in examining such engagements stems from a perspective of biological associations, namely the phenomenon of symbiosis. My preoccupation with symbiosis is fuelled in equal parts by the phenomenon's scientific significance towards evolution, and the way it has informed seminal philosophical observations on social organization among humans [1]. The outcome of this artistic research resulted in two publications $[2,3]$ with symbiosis informing a framework through which I went to analyze the process of and the social dynamics observed within the collaborative engagements conducted as part of my practice, largely concerning live performance works involving interaction between music and dance. At the same time, however, these publications provided scarce details on the technological means used to facilitate the interaction between our respective expressive media. Considering the fundamental role of technology in developing our joint practice outcomes, further reflection on the conceptual debate between symbiosis and performance practice complemented the existing framework for interaction between disciplines and practitioners resulted in findings that appear salient towards identifying distinct modes of interaction between the expressive media of sound and movement.

The findings presented in this chapter have been reached through a Practice Research methodology, and are presented through a process of analytic autoethnography, that is the active reflection of one's experiences in creative work through the wider context of associated practices. The contribution of these findings on technology focus on the application of well-established GRT devices and associated software suites within a novel set of interaction modes informed by the taxonomy of symbiotic relationships, with the activation of these modes presented through both precedent examples, as well as a representative outcome developed through my collaborative practice. In other words, rather than aiming towards creating new technologies for creative expression, this research aims to present new ways of appropriating existing technologies, particularly in manners accessible to practitioners of diverse levels of technological proficiency. 
My motivation towards this research approach stems from my previous practice as a performer of Electronic Dance Music, where the limited possibilities presented by analogue disc jockey equipment were a driving force to develop new ways for sonic manipulation. This exploration continued during my first steps within sonic arts, albeit this time through predominantly digital tools, with the addition of modulation devices (e.g. Envelope Generators (EG), Low Frequency Oscillators (LFO), Step Sequencers), as means of predefining and accurately recreating certain sonic gestures in the performed material. However, the accuracy of digital devices made away with the organic and ephemeral feel borne of the imperfections of human movements. This observation coincides with my first forays into collaboration with dancers, as well as my initial experimentations with GRT in sonic performance through the widely used Nintendo Wii Remote (or Wiimote). These early experiments were conducted in collaboration with contemporary dancer Shona Roberts, an already close personal friend, with whom we began exploring ways for motion sensors to manipulate and modulate sound (see Fig. 8.1).

At the time, my main focus was to explore the potential of using physical movement as means of affecting, guiding, and enriching sound, with the connection between the two expressive media being a lesser concern. In essence, my perspective at the time could be described as sonic-centric, where the performer's actions were utilized as merely another source of modulation to augment the previously used devices. Contrary to reducing my collaborator's contributions to the programmed actions of a machine, this approach allowed Roberts unrestricted range of expression

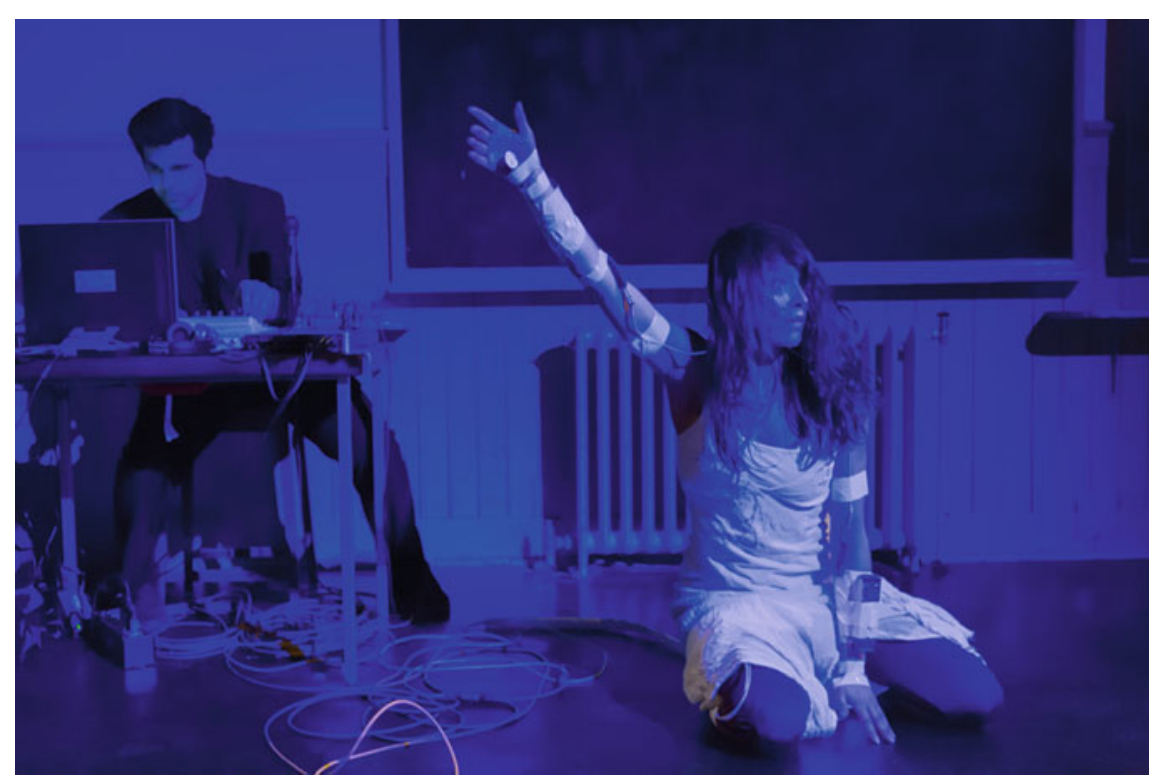

Fig. 8.1 Performance with Shona Roberts at Anatomy, Edinburgh Summerhall, April 2014 (Copyright Richard Dyson, reproduced by permission) 
through her choreography, while I enjoyed similar liberty in creating sonically meaningful interpretations of the dancer's arm movement through the data captured by the pair of Wiimotes.

\subsection{Interactivity in Performance-Origins and Recent Developments}

On reflection, this mutual independence during our earliest experiments with Roberts echoed the collaborative relationship between composer John Cage and choreographer Merce Cunningham, who in developing their joint works would 'intentionally segregate the creation of the sound from the creation of the movement until the performance' [4]. Dubbing this relationship 'autonomous complementarity', Andrew Uroskie explains that this separate development of disciplinary material was an effort in alleviating the dependency of movement to embodying sound in a manner that was to an extent literal, and certainly perceivable by the audience:

Music was understood to govern, implicitly or explicitly, the movement of the dancers.

Choreography, within this propulsive conception, was a kind of musical interpretation, judged

on its ability to form a singular synaesthetic coherence in the experience of the audience. [5]

Challenging the traditional notions of audience experience in the work of Cage and Cunningham is further evident from the presentation format of the Black Mountain 'Happenings', a series of events where the combination of music, dance, and visual arts was such as to allow each audience member to experience a unique perspective $[5,6]$. The two artists' focus was shifted from the experience of the audience to that of the performers, and the manner in which the latter embodied the received aural and ocular stimuli.

Having explored independence between sound and movement throughout their work in the 1950s, in 1965 Cage and Cunningham developed Variations V, arguably the first performance to feature technologically facilitated interaction between music and dance. Working alongside the artists, engineers Billy Klüver and Robert Moog developed two distinct approaches in motion-activated control of music equipment. Respectively employing photosensitive cells and proximity antennas [7], the system was designed as to react to dancers' movements by operating the transport controls of several tape machines storing Cage's sound palette. As such, the notion of sound governing the 'propulsion' of movement was all but eliminated in Variations $V$. However, as Uroskie points out, the dancers were not in conscious control of the sonic palette, which would imply that 'one model of subordination would have merely been exchanged for another'; instead, movement was utilized to 'set a certain train of sonic events in motion' [5]. In other words, while dancers performed Cunningham's precisely rehearsed choreography [7], the sounds triggered from the interaction between movement and the two motion-detecting systems resulted in an indeterminate sonic outcome, an approach in music score that is often associated with the practice of Cage. 
With the benefit of more than fifty years of technological developments, a modern look on the modes of interaction between sound and movement in Variations $V$ could deduct that the artists were not able to implement a precise control of sound through movement due to the limitations of the relatively crude analogue system in interpreting movement into actuating commands. Nowadays, interpreting movement into digital data has given rise to GRT devices that allow a detailed level of controlling software through physical movement. Such systems are implemented in diverse applications within the fields of computing, manufacturing, as well as arts. On the latter field, researchers and practitioners exploring interaction between sound and movement have formed dedicated communities, such as the annual conference New Instruments for Musical Expression (NIME).

While much of the focus of this research field has been the development of new technologies and methodologies facilitating the expression of sound through movement, implementing these contributions demands a certain level of technical proficiency and knowledge of the associated language and vocabulary. In my practice, I was faced with this issue during my early collaborations with Roberts. To further contextualize the aforementioned independence between our respective material, our approach resulted from the lack of a shared vocabulary. In other words, while Variations $V$ presupposed a degree of freedom between sound and movement due to imprecise technology, that freedom in my work with Roberts was dictated due to imprecise language. Although my mapping of the Wiimotes' motion data to sound processing parameters made sense to practitioners familiar with audio software, communicating these mappings verbatim to my collaborator was inefficient in allowing Roberts to understand how her movement affected sound, an issue further compounded by my own lack of knowledge in the language of choreography. While we were able to overcome these issues due to our prolonged collaboration and personal familiarity, I was aware that the privilege of time and patience will not always be present. This realization prompted me to explore a way of communicating my desired intentions in shaping sound through movement via the use of GRT. Prior to this specific research question, my research contributed a framework comprising of a set of strategies and precepts towards guiding polydisciplinary collaborative process [2], with its core concept being the biological phenomenon of symbiosis. From its serendipitous appropriation as the name of our initial collaborative engagement, examining the scientific research of the phenomenon in the context of artistic practice allowed for the development of a system derived from a disciplinary-neutral field, thus avoiding presupposing greater importance to neither of our disciplines.

\subsection{The Symbiotic Phenomenon}

The lexicological definition of symbiosis (Oxford English Dictionary) suggests two elements existing in a sustained harmonious relationship. However, in the context of biological associations, harmonious coexistence is merely one of the many manifestations of symbiotic relationships. Biologists define symbiosis as the close 
and persistent relationships between organisms of different species [8]. Organisms engaged in symbiosis are identified as the host and its symbiont, with the engagement most often initiated by the symbiont becoming attached to the typically larger host, motivated by the former's desire to extract benefit from the relationship. In line with the different levels of extraction of benefit, or fitness outcome [9], symbiosis manifests in a variety of types. The three core types are mutualism, commensalism, and parasitism, with each type identified according to the effects it has on the engaged organisms. And since the symbiont is always benefited, it is the effect on the host which defines the type (see Table 8.1).

This taxonomy of relationships refers to symbioses as they are observed at a specific moment in time, described as research on ecological scale [10]. However, examining symbiosis over longer timescales reveals its function as an evolutionary mechanism, with the organisms' evolutionary trajectory reacting to the close and persistent interactions with their partner's specific traits. For example, while a particular partnership may have begun as parasitic (which is often the origin of most interspecific associations [9]), the host will eventually evolve ways to extract benefit from the relationship, while the symbiont will also manage its exploitation of the host with the aim of prolonging the relationship. This reactive evolution serves as proof for an evolutionary trend from parasitism to mutualism, which is nowadays widely accepted by biologists [10].

Having explained the core taxonomy of symbiotic relationships, the next step towards drawing parallels between biological and creative associations is to firstly identify the elements making up each partnership, and secondly establish a relationship between these elements. The first shared aspect among the two forms of association concerns the partners' motivation towards establishing a relationship; that is to combine their individual traits as means of jointly overcoming limitations, respectively borne of environmental [8] and disciplinary [11] factors. From this starting point, the remaining elements are placed through a process of conceptual debate, and finally become organized opposite each other, as summarized in Table 8.2.

This subjective interpretation assumes the symbiotic relationship as the collaborative engagement, with the organisms as the collaborating practitioners. The interspecificity of the engaged organisms is reflected through the different disciplines employed by each practitioner, with each discipline's specific expressive media related to the biological traits carried by each species. In interpreting the roles of host and symbiont, these are allocated respectively on the practitioner instigating the collaboration and the one who is guided according to the former's direction. As will become clearer later on, the used nomenclature applies to engagements which

Table 8.1 Symbiosis typology and fitness outcome

\begin{tabular}{l|l|l}
\hline \multirow{2}{*}{ Type of interaction } & Fitness outcome \\
\cline { 2 - 3 } & Symbiont & Host \\
\hline Mutualistic & Positive & Positive \\
\hline Commensalistic & Positive & Neutral \\
\hline Parasitic & Positive & Negative \\
\hline
\end{tabular}


Table 8.2 Related elements of symbiotic relationships and polydisciplinary collaborations

\begin{tabular}{c|c|c|c}
\hline Symbiotic relationships & Polydisciplinary collaborations \\
\hline Organisms & Symbiont & Instigator & \multirow{2}{*}{ Practitioners } \\
\cline { 2 - 3 } & Host & Directee & \\
\hline Interspecificity & Biological traits & Expressive media & Polydisciplinarity \\
\hline \multicolumn{2}{c}{ Fitness outcome } & \multicolumn{2}{|c}{ Expressive range } \\
\hline
\end{tabular}

Fig. 8.2 Effect direction in the different types of symbiosis and collaborative process

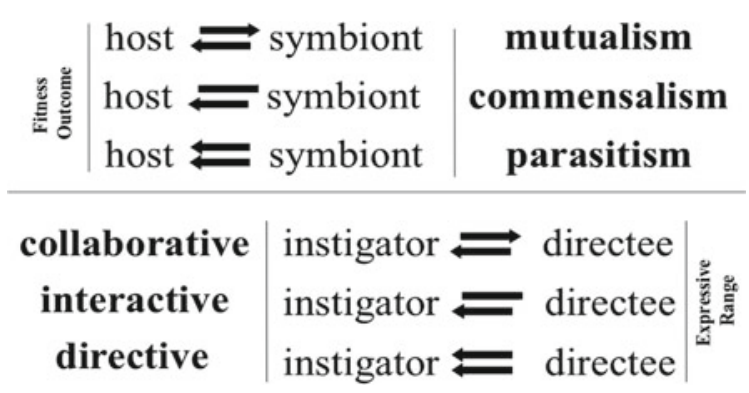

feature a higher level of dependency, be that a parasitic relationship in symbiosis or a directive collaboration [12] (see note 2) in artistic practice, with the prescriptive meaning of these roles diffused during commensalistic/interactive engagements, and almost entirely absent in mutualistic relationships and collaborative modes.

The final interpretation concerns the element of fitness outcome into expressive range. As mentioned, the types of symbiotic relationships are identified according to the host's fitness outcome, or the level of benefit that organism experiences as a result of its engagement with the symbiont. In the context of my interpretation, expressive range refers to the level of creative input allocated to each practitioner during their collaboration (see note 3 ). Figure 8.2 showcases the shared direction of effect between the two types of association, with the partner' extraction (or reciprocal exchange) of fitness outcome in symbioses, and delimitation (or conversation) of expressive range in collaborations. In the context of polydisciplinary collaborations, this approach presents an efficient way of understanding and allocating each practitioner's liberty in developing their respective disciplinary material during the process of collaborative engagements (see note 4), with the same principle available towards organizing the relationship between musician and dancer while operating an interactive system.

\subsection{Interactive Taxonomy: Theory and Precedents}

As mentioned in the introduction, the typical approach of using GRT in musicdance interaction concerns the change of sound through movement. This effect is achieved by mapping movement data to various parameters of DSP devices, with the 
sound consequently affected as a result of the movement data performing alternations on the parameter's values. Considering this relationship between the two media, sound can be understood as the symbiont medium, with movement being the host governing the development of sound. With this principle in place, and taking into account a host's different fitness outcomes during each type of symbiosis, an equal number of interaction modes can be derived, where the 'host' movement can be 'benefited', 'harmed', or 'unaffected' by its 'symbiont' medium of sound. In line with the previous subjective interpretation between the elements making up each association, the biological notions describing changes in fitness outcome are related to the restrictions, or lack of, placed on the expressive range of the associated media and their respective practitioners during a performance. The most efficient way to establish these relationships is by first observing the resulting sonic outcomes, followed by the restrictions placed on the movement, the provision in which the two performers develop their respective sonic and movement material, and finally the dancer's awareness of how their movement affects sound while operating the system.

Looking at the first association, when the collaborative performance requires a determined sonic outcome (akin to a fixed music score), the dancer must perform a specific set of movements in order to alter the values of the DSP parameters in a predefined manner. As a result, this interaction mode imposes restrictions on the movement's range of expression in order to accommodate the desired sonic outcomes. Furthermore, with the mappings between movement data and DSP parameters having been created by the music practitioner, she or he needs to communicate to the dancer the required movements needed to achieve the determined development of sound over the duration of the performance. Consequently, the dancer is relieved from having to fully understand the ways her or his movements may affect sound beyond the predefined movements. As such, this interaction mode assumes movement as a predefined modulator for the sound. In other words, through the previously discussed subjective interpretation of the biological notions describing fitness outcome, the 'host' movement is 'harmed' in order to 'benefit' its 'symbiont' sound, thus establishing a parasitic symbiosis between the two expressive media.

On the opposite spectrum of sonic outcome, an indeterminate score entirely alleviates any requirement for the dancer to become familiar with the mappings between movement data and DSP parameters, with movement remaining independent to sound. However, from the musician's perspective, the mapping must be designed as to accommodate the dancer's full range of movements which she or he may deploy in any manner and time throughout the duration of the performance. In a way, the randomized alteration of DSP parameters during this interaction mode can be related to a generative music system, or to provide a further simplified reflection, to the modulations derived by an LFO set to random or noise waveform. In the context of the symbiotic interpretation, the 'host' movement is 'unaffected' due to enjoying a full range of expression, while the 'symbiont' sound extracts 'benefit' in the form of randomized modulations that can be used to develop and expand its outcome. As such, this interaction mode forms a commensalistic symbiosis between sound and movement, which on reflection can be associated with the interaction employed in 
Variations $V$, when dancers had an effect on sound despite being unaware of the ways their movements specifically controlled Cage's tape players. However, while Cage revelled in employed indeterminacy as a compositional approach, Cunningham directed his dancers through an explicit choreography. Nevertheless, this is but one manifestation of a commensalistic interaction mode, and as I present later in this chapter, free improvization presents itself as fruitful provision for the dancer to follow, with the musician tasked with designing a system able to generate meaningful sonic outcome through random modulation inputs.

With mutualism being the remaining type of symbiotic relationship, such an interpretation into the context of collaborative performance requires for both sound and movement to mutually extract 'benefit' from their interaction, which considering the earlier connection between fitness outcome and expressive range, suggest a simultaneously full range of expression for both media. While the provision of free improvization may at first appear salient to this mode, developing this mode through practice showcased that an intermediate provision is more appropriate, that of structured improvization. Examining this provision in the context of music performance, structured improvization differs from its free counterpart by the approach of creating real-time compositions by connecting pre-established material over an arrangement which is not predefined [13]. As such, while the resulting sonic outcome is not determined, its characteristics can be anticipated. Structured improvization shares a slightly different meaning in the context of choreography, with dancers adhering to a predefined temporal arrangement in relation to stage placement and clustering, while retaining freedom towards their performed movements during each section of the arrangement [14]. Considering these provisions for music and dance respectively, in the context of GRT-facilitated interaction the dancer is allocated freedom towards her or his movements, with the caveat that these movements need to result to anticipated sonic outcomes. As such, the dancer must be well-familiarized with the system's mapping, and be aware of the ways each movement may affect sound. In other words, the mutualistic interaction mode presents a mutual compromise between the expressive ranges allocated to sound and movement, with both media mutually extracting 'benefit' up to the level at which one of them can be said to experience 'harm', thus resembling the mechanisms by which mutualistic symbioses are developed over evolutionary scale in the natural world.

The three symbiotic modes of interaction are summarized in Table 8.3, with each mode identified according to their specific effect awareness, provision,

Table 8.3 Taxonomy of symbiotic interaction modes with associated elements

\begin{tabular}{l|l|l|l}
\hline \multirow{2}{*}{ Affect awareness } & Interaction modes & \multicolumn{2}{|l}{} \\
\cline { 2 - 4 } & Mutualism & Commensalism & Parasitism \\
\hline Provision & Structured improvisation & Free improvisation & Score/choreography \\
\hline Operation & Exploration & Detachment & Instruction \\
\hline Outcome & Anticipation & Indeterminacy & Determinacy \\
\hline
\end{tabular}


operation, and outcome borne of the interaction between the two media. At this stage, it is worth pointing out once again the subjectivity and conceptual nature of this interpretation, and furthermore the Practice Research methodology employed towards reaching these findings, with the latter derived through the accumulated knowledge from numerous years of collaborative practice alongside several practitioners. As presented later in this chapter through the work Symbiont Zero [15], in addition to employing distinct modes of interaction, multiple modes can also manifest during a performance, either consecutively during different sections, or simultaneously while operating different layers of sound, each controlled via a different mode. Prior to describing our collaborative outcome and designed interaction system, I demonstrate the taxonomy's theoretical standing by examining three interactive works as means of identifying their specific modes of interaction. Rather than providing a conclusive literature review of the field's practical outcomes, the presented precedents were selected as to represent distinct uses of GRT, collaborative processes, and employed aesthetics.

\subsection{Symbiotic Interactions in Precedent Practices}

Stratofyzika is a Berlin-based collective, with its founding member comprising musician Lenka Kocisova, visual artist Alessandra Leone, and dancer Heather Nicole (Hen Lovely Bird). Their 2016 work THAETA [16] presents a dialogue between the three media of sound, movement, and visual. In the context of the symbiotic concept, the dancer serves as the host modulating sound and visuals, with the interaction facilitated through a wearable Inertia Measurement Unit attached on the dancer's arm, with the movement data simultaneously modulating the two software suites generating sound and visuals. According to Leone, rather than having a constant flow of movement data into the system, this flow is interrupted by altering the correlation between movement data and resulting value alteration via algorithms [17], as well as activating the data stream only during predefined moments. With the dancer aware of the way her movement affect the other two media, it is during those moments of activated interaction that the dancer is able to freely employ movements that result anticipated sonic and visual outcome, such as the floor roll (see [16], 1:10-1:20) resulting in a white spire and a tone of descending pitch.

The second precedent is one of the audio-visual installations developed with the danceroom Spectroscopy $(\mathrm{dS})$ visualization system. Using an array of depth perception cameras (Microsoft Kinect) as its GRT devices, the system is able to extract movement data from multiple participants, with the date used to modulate the visualizations. While dS was designed primarily as a research tool for molecular dynamics, allowing researchers to visualize particle movement, as well as interact with their visual representations [18], it then became the basis for a series of creative outcomes with the addition of a sound-generating system sharing the data modulating the visualizations, with the movement data coming from a troupe of dancers. In addition to the performances, the team behind $\mathrm{dS}$ also presented the system in the 
configuration of a public multi-participatory installation, where audience members are invited to interact with the system. The result of this interaction is that sonic and visual events are manipulated by movement without the controllers having any prior training with the system [19], thus demonstrating a commensalistic interaction mode. A point of interest here is that once an audience member began exploring the system, they would attempt to understand what their effect is on the media, resulting in an adaption of the interaction mode. While this does not in itself form an undesirable aspect, I later present a solution devised towards maintaining commensalism during interaction.

Finally, as an example of practices displaying parasitic interaction between sound and movement, Marco Donnarumma's Corpus Nil [20] is a work which appears to absolutely appropriate the notion. For this work, Donnarumma devised a choreography comprising of 'five key bodily postures' [21], each designed as to force the system to generate a specific response from the sound and lights it controls. With the system operating through machine learning algorithms able to identify specific positions by their duration, the performer must sustain these positions as to progress through the arrangement. As such, Donnarumma is restricted by the design of these key positions, or more accurately, is able to move only within the restrictions posed by each position. Understanding this approach through the symbiotic framework, the performer possesses a detailed awareness of the ways their movement affects sound and lights, and is able to perform the determinate sonic outcome through their movements. This sits in contrast to the approach employed by Stratofyzika and danceroom Spectroscopy; in the latter, audience members are unaware of the ways the system interprets their movements as modulations for the other two media, whereas in the former, the dancer is aware of the effects her movements have on the interacting media, and is allowed to explore movements along with the effects these have on sound and visuals. In the case of Corpus Nil, the performer's movements are intended to achieve predetermined changes to the other two media.

\subsection{Symbiotic Modes of Interaction in Symbiont Zero}

As mentioned earlier, the findings presented in this chapter stem from my collaborative practice alongside several dancers. Following the development of the theoretical frameworks for polydisciplinary collaboration and symbiotic modes of interaction, my aim was to activate the entire spectrum of interaction modes within a single creative outcome, with the additional purpose of serving as a demonstration of the framework. In order to satisfy both purposes, Symbiont Zero has been documented both as complete performance, as well as a set of demonstration videos, with the latter displaying a simplified version of each interaction mode, with additional perspectives providing simultaneous views of the musician's inputs via external controllers, as well as the effects both performers conduct on the mapped parameters (see Fig. 8.3 for a representative example). 


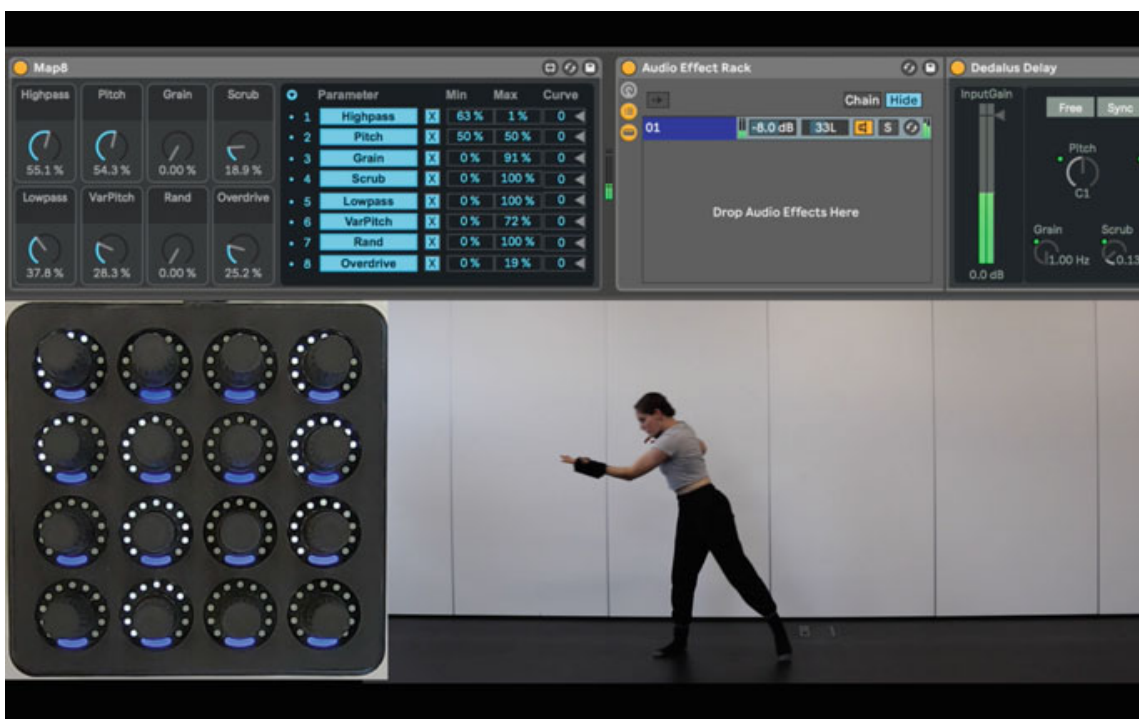

Fig. 8.3 Demonstration video with multiple views: dancer (Cunliffe), external controller, and mapping distribution matrix

The work was initially developed with my long-term collaborator Roberts, and subsequently performed with contemporary dancers Lucie Lee, Joseph Lau, and more recently Kelsea-Leigh Cunliffe. The premise concerns a musician-dancer duet featuring interaction between their respective expressive media through GRT. With a total duration of approximately fifteen minutes, the piece develops over three movements, or sections, of equal duration, and while each section predominantly focuses on a single mode of interaction, the performers have gone to implement areas of adaptation through simultaneous employment of different modes while controlling multiple layer of sound.

In describing the path of the movement data into the system, the concerned GRT devices comprise a pair of Wiimotes tethered on the dancer's forearms as means of performing alterations to the sound-generating system. This system is hosted within Ableton Live, receiving a total of eight data streams from the two Wiimotes, following their capture and conversion into MIDI CC messages through the application OSCulator. These continuous messages are then mapped onto two Max for Live devices, namely, Map8 and Multimap, which serve two purposes; firstly, acting as a distribution matrix from which to map each data stream onto multiple DSP parameters, and secondly, allowing the musician to toggle the activity of each mapping, as well as alter the range, direction, and curve of each mapping's effect on their destination during the performance. The musician's inputs are achieved through two hardware MIDI controllers combining continuous and toggle controls. The data path is graphically represented in Fig. 8.4, (albeit for the data from a single Wiimote 


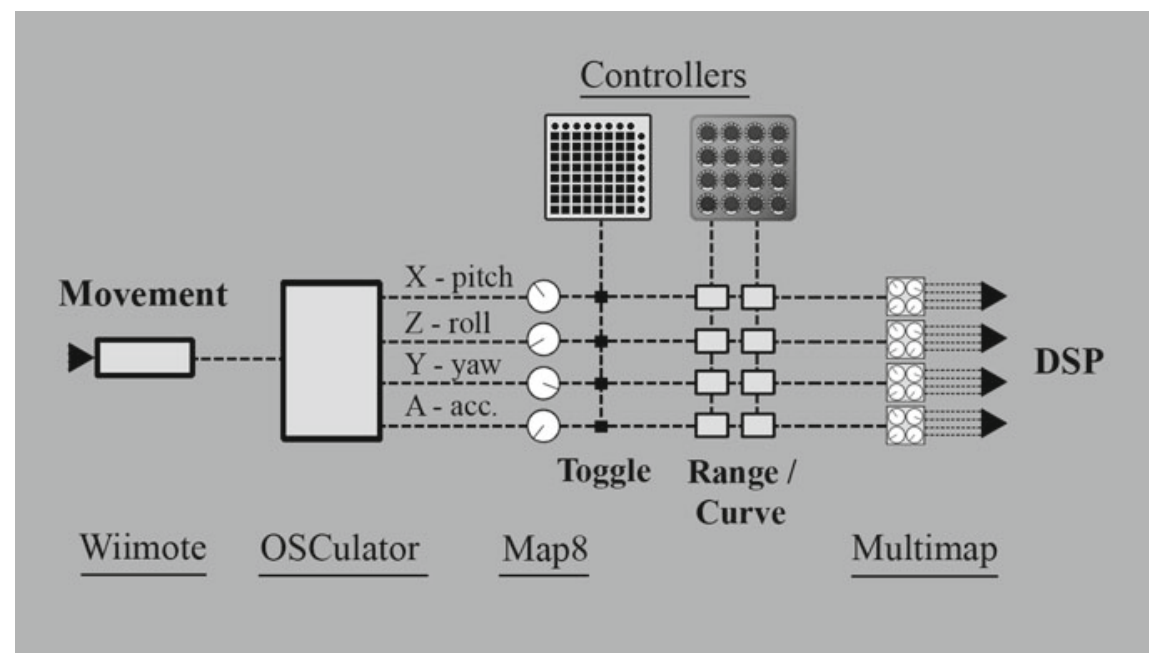

Fig. 8.4 Path of gesture data, from dancer's movement to DSP mapping

only), with the following subsections describing the work's three sections in terms of both system operation and performance planning. Each section is described under the title of their predominant mode of interaction.

\subsection{Mutualism}

In line with the aspects of mutualistic interactions, the dancer has been familiarized with the system, and is able to anticipate the ways her movements may affect sound. Furthermore, the performers have noted certain combinations of movements and specific ranges in the distribution matrix which resulted in interesting sonic outcomes, which the performer has memorized and able to deploy at will.

The main interactive sound during the mutualistic section is generated by a feedback delay (Amazing Noises Dedalus Delay), with its parameters mapped to the Wiimotes' eight continuous messages, these being X-axis (vertical position), Y-axis (roll), Z-axis (yaw), and acceleration. In this case, the $\mathrm{X}$-axis of both devices is assigned to the delay's two filters, low pass and high pass for left and right arm, respectively. This particular mapping ensures that the performer maintains control over the overall signal amplitude, which subsides by simultaneously pointing both arms upwards due to the removal of high or low frequencies by either filters. The remaining mappings are arranged to parameters such as pitch shifting, size and frequency of grain, and addition of parallel distortion and reverberation.

The musician's role during this section is to adjust the range by which each continuous message affects the mapped parameters. This is achieved by adjusting the range parameters on the Map8 device, thus altering the dancer's level of influence 
on each mapped parameter. The section begins with the filter ranges restricted middle frequencies, while the dancer concentrates on upper body movements. Halfway through the section, the musician broadens the range of the filter mapping, causing a sudden increase in amplitude. This serves as a signal for the dancer to initiate weight transfer movements, resulting longer movements across the stage.

\subsection{Commensalism}

As described in previous sections, commensalistic interactions alleviate the need for the dancer to possess prior knowledge of the ways movement affects sound. However, as mentioned in the description of $\mathrm{dS}$, participants in control of sound will naturally begin to discover the connections between their movements and changes in sound, resulting in an adaptation of mode. Aiming to avoid this adaptation during the commensalistic section of Symbiont Zero, I designed a system able to dynamically alter the mapping during the performance. This is achieved by mapping a single movement stream of continuous change message to several DSP parameters by inserting an instance of Multimap between one of Map8's outputs and the DSP inputs. Furthermore, toggles are assigned to toggle each of the Multimap's outputs. The result of this design allows the musician to continuously alter the movement data stream distribution among the mapped DSP parameters, and thus avoid the dancer to gain a fixed relationship between her movement and specific sound modulations.

The sound generation is based on a series of granulators (Audiority Grainspace) processing recordings of synthesized percussive patterns, with the Wiimotes' CCs mapped to parameters such as start-end of input sample, pitch and tone variations, saturation, size of and distance between grains, and level of parallel reverberation.

While the dancer is entirely at liberty to freely improvise her movements, she is also motivated to 'forget' the interaction, and instead treat the resulting sound as recording, akin to improvising to a fixed (non-interactive) composition. The only cognisant interaction of the performer occurs after a new layer of sound appears, made of two percussive samples (respectively kick drum on the left arm and snare drum on the right arm), each activated by the acceleration streams going over a set threshold which triggers a MIDI Note on message mapped to an instance of Ableton's Drum Rack containing the percussive samples.

Once the performer becomes aware of the new layer, she is able to interact with the two sounds through a mutualistic mode, while simultaneously maintaining her commensalistic interaction with the previous sound layer. The section ends with the initiation of a further sound layer made of arpeggiated synthesiser pattern, signifying the transition into the final section. 


\subsection{Parasitism}

The parasitic interaction presents the highest level of accuracy from the dancer, while at the same time imposes the highest level of restriction on their movements. The Wiimotes' CCs are mapped to a synthesiser (Ableton Analog) generating a fixed arpeggio pattern, with the left arm controlling the synthesiser's low pass filter cutoff frequency on the $\mathrm{X}$-axis and envelope attack duration on the $\mathrm{Y}$-axis (rotation), while the right arm controls reverb depth and delay feedback on X-and Y-axis, respectively. In line with the approach during parasitic interaction modes, the sonic outcome is determined, in this case being a slow modulation between the wet-dry balance of the reverb, followed by a filter frequency sweep of equal duration. At the same time, the determined outcome requires for staccato (sudden jumps in value) modulations on the two remaining parameters, resulting in altering the note's envelope shape and feedback level. Finally, the two percussive samples from the previous sections are required to be activated at the end of each filter sweep cycle, resulting in the dancer interacting with this layer through a parasitic mode, as opposed to the previous mutualistic engagement.

With the determined sonic outcome providing a limited range of movements for the dancer, the latter is then free to develop their own choreography within this restricted range. Having performed the piece with four different dancers, a particularly interesting finding is that while each dancer were able to achieve the determined sonic outcome, each reached that through distinct sequence of movements. For example, Roberts maintained her core still while only moving her arms (as to achieve the modulations on the synthesiser's parameters), Lee continued to move across the stage while limiting the movement of her arms in line with the score. Similarly, while Lee activated the percussive samples by performing waving movements with her arms, Cunliffe opted to perform sudden contractions and extension, akin to punching motions. This showcases that despite the imposed restrictions during parasitic interactions, dancers are able to interpret the score through different choreographic movements.

In describing the remaining section, once the filter sweep sequence has been performed twice, the dancer is instructed to resume a mutualistic interaction with the percussive samples, while at the same time the musician limits the mapping range of the synthesiser parameters, who is then able to assume control of these parameters through their external controller. As a result, the musician 'takes' away control from the dancer while she breaks free from the previous restrictions. This continues for around one minute, before the dancer assumes the previous position, and the musician returns the control of the synthesiser back to the dancer, who repeats the previous movements twice. Symbiont Zero concludes with a short section of commensalistic interaction, where the performer's movements influence a pair of granulators (New Sonic Arts Granite) processing two segments of recorded music, namely, the 'Amen' and 'Hot Pants' drum breaks. As with the previous section, the performer is unaware of the effect their movements exert on the newly introduced sounds, and is instructed 
to perform an improvized sequence, with its intensity influenced by the overall level of sound made of the combined elements, which begins to fade until silence to the end of the piece.

\subsection{Conclusions}

Although the analyzed precedents do not constitute an exhaustive review of the field, the interactive works portray a selected collection of practices displaying diversity in GRT (Inertia Measurement Units, depth perception cameras, and biophysical sensors), in the make up of their collective creation (inter/multi/trans-disciplinary respectively), and as explained earlier, in the employed interaction mode between physical movement and digital audio-visual material. Appropriating the typology of symbiotic relationships towards forming a new taxonomy in technologically facilitated interaction allows for the diversity in which interactive performances manifest to be identified within just three modes of interaction, each comprising of thee aspectsprovision, operation, and outcome — as well as the level of training or familiarity with the system required by the dancer. This serves as an efficient approach towards communicating the purpose of each mode, particularly assisted by the lack of convoluted terminology, which can often be counterproductive when used to communicate the desired outcome to practitioners whose expertise focus on other areas of practice.

This research has thus far focused on dyadic collaboration, with future view of assessing the symbiotic concept with multiple partners. Furthermore, while the use of consumer GRT devices was implemented as means of making the research outcomes more inclusive (due to being both reliable and inexpensive), the limited accuracy and expansion of these devices have become to emerge in my practice, which serves as motivation to look into developing bespoke devices, in line with current trends in the field of interactive arts research.

Most importantly, however, it is my strong belief that the future of interactive arts will greatly benefit from researchers combining technological innovation with artistic Practice Research, a symbiotic relationship if you will. In my experience, although some steps are already taken by few brave individuals, the two fields' distinct approaches, cultures, sensitivities, and nomenclature result in hesitation towards tighter integration. But akin to the parasitic beginnings from which most symbioses begin their evolutionary journey, persistence and closeness will eventually manifest in mutual aid among the two domains of creative practice, artistic and scientific. In the words of Pëtr Kropotkin, 'In the practice of mutual aid... we thus find the positive and undoubted origin of our ethical conceptions; we also see the best guarantee of a still loftier evolution of our race' [1]. 


\section{Notes}

(1) The term polydisciplinary is used to denote the multiple modes by which disciplines interact, as an alternative to the more popular 'interdisciplinary', which I consider to be erroneously habituated for this purpose, as it ignores established principles of taxonomy. I provide a more thorough discussion on this subject in Sect. 2.2.2 of [3].

(2) The referenced modes of collaboration are derived from the work of Hayden and Windsor [12], identified as directive, interactive, and collaborative, each suggesting a different level of creative liberty between the engaged practitioners.

(3) The social aspects of collaboration, along with the notions of allocating creative direction duties, privileges, and responsibilities, have been addressed in my previous paper [2].

(4) For a detailed description of the symbiotic framework on collaborative process, see chapter three of [3].

\section{References}

1. Kropotkin, P.: Mutual Aid: A Factor of Evolution. McClure Phillips \& Co., New York (1902)

2. Moriaty, M.: Symbiosis: organisation and mutual exploitation in interdisciplinary collaboration, artistic research will eat itself. In: Proceedings of The 9th SAR International Conference on Artistic Research, University of Plymouth, UK, 11-13 Apr 2018. Cox, G., Drayson, H., Fatehrad, A., Gall, A., Hopes, L., Lewin, A., Prior, A. (eds.): Society for Artistic Research, pp. 81-96 (2018)

3. Moraitis, E. (Moriaty, M): Symbiotic synergies: adaptive framework for polydisciplinary collaboration in performance practice. Ph.D. thesis, School of Arts \& Media, University of Salford, Manchester (2019)

4. Klosty, J.: Merce Cunningham. E.P. Dutton, New York (1975)

5. Uroskie, A.V.: from pictorial collage to intermedia assemblage. Animat. Interdiscip. J. 5-2, 223-241 (2010)

6. Kirby, M.: Happenings: An Illustrated Anthology. R\&R Books, Reno (1966)

7. Miller, L.E.: Cage, Cunningham, and Collaborators: The Odyssey of Variations V. The Musical Quarterly, Oxford University Press, vol. 85-3, pp. 545-567 (2001)

8. Douglas, A.E.: The Symbiotic Habit. Princeton University Press, New Jersey (2010)

9. Paracer, S., Ahmadjian, V.: Symbiosis: An Introduction to Biological Associations. Oxford University Press, New York (2000)

10. Sapp, J.: Evolution by Association: A History of Symbiosis. Oxford University Press, New York (1994)

11. John-Steiner, V.: Creative Collaboration. Oxford University Press, New York (2000)

12. Hayden, S., Windsor, L.: Collaboration and the composer: case studies from the end of the 20th century. 61-240, 28-39. Cambridge University Press, Tempo (2007)

13. Cage, J., Kirby, M., Schechner, R.: An interview with John Cage. Tulane Drama Rev. 10-2, 50-72 (1965)

14. Pressing, J.: Improvisations, methods and models. In: Sloboda, J. (ed.) Generative Processes in Music, Clarendon Press, New York, pp. 129-178 (1987)

15. Moriaty, M.: Symbiont zero: multimodal interactive performance, 19 Aug 2019. Accessed 20 Nov 2019 [Online]. https://manolimoriaty.com/symbiont-zero/ 
16. Leone, A.: StratoFyzika-THÆTA, 16 Dec 2016. Accessed 20 Nov 2019 [Online]. https:// vimeo.com/195961322

17. Leone, A.: Personal interview, 19 Feb 2018

18. Glowacki, D.R., O'Connor, M., Calabró, G., Price, J., Tew, P., Mitchell, T., Hyde, J., Tew, D.P., Coughtrie, D.J., McIntosh-Smith, S.: A GPU-accelerated immersive audio-visual framework for interaction with molecular dynamics using consumer depth sensors. Faraday Discuss 169, 63-87 (2014)

19. Royal Society of Chemistry: Atoms and molecules are not static by dance room Spectroscopy (dS), 3 June 2014 [Online]. https://www.youtube.com/watch?v=H_EJquvSBMQ. Accessed 20 Nov 2019

20. Donnarumma, M.: Corpus Nil, 22 Jan 2016 [Online]. Accessed 20 Nov 2019 [Online]. https:// vimeo.com/152710490

21. Donnarumma, M.: Configuring corporeality: performing bodies, vibrations and new musical instruments, Ph.D. thesis, Department of Computing, Goldsmiths University of London, London (2016)

Open Access This chapter is licensed under the terms of the Creative Commons Attribution 4.0 International License (http://creativecommons.org/licenses/by/4.0/), which permits use, sharing, adaptation, distribution and reproduction in any medium or format, as long as you give appropriate credit to the original author(s) and the source, provide a link to the Creative Commons license and indicate if changes were made.

The images or other third party material in this chapter are included in the chapter's Creative Commons license, unless indicated otherwise in a credit line to the material. If material is not included in the chapter's Creative Commons license and your intended use is not permitted by statutory regulation or exceeds the permitted use, you will need to obtain permission directly from the copyright holder. 